\title{
ON A PROBLEM OF MONTGOMERY
}

\section{CHUNG-TAO YANG}

1. Let $M$ be a manifold and let $G$ be a compact Lie group acting on $M$. For $x \in M, G_{x}$ denotes the isotropic subgroup of $G$ at $x$, that is, the subgroup consisting of all the elements of $G$ which leave $x$ fixed. Then the following problem has been raised by Montgomery (see Ann. of Math. (2) vol. 50 (1949) p. 260): Do there exist a finite number of subgroups of $G$ such that each $G_{x}$ is conjugate to one of them? The purpose of this note is to show that, if $M$ is compact and differentiability is assumed, then the answer is in the positive. Whether this result remains true without the assumption of differentiability is still unsettled. But if $M$ is not compact, it is known that even for the analytic case the answer is in the negative. We shall give below an example to this effect, which was communicated to the author by Professor Montgomery.

2. Theorem. Let $M$ be a differentiable manifold and let $G$ be a compact Lie group which acts differentiably on $M$. Then for each point $p$ of $M$ there exists a neighborhood $U$ of $p$ and a finite number of subgroups of $G$ such that the isotropic subgroup of $G$ at any point of $U$ is conjugate to one of these subgroups.

Proof. The proof of this theorem relies on a lemma by Montgomery, Samelson and the author (see Ann. of Math. (2) vol. 64 (1956) p. 136), which says: If $M$ and $G$ are as in the theorem and $p$ is a point of $M$, then there is a closed cell $K$ in $M$ and a closed cell $Q$ in $G$ satisfying the following conditions:

(i) $K$ contains $p$ and $Q$ contains the identity of $G$.

(ii) Whenever $g \in G$ and $x \in K, g(x) \in K$ if and only if $g \in G_{p}$.

(iii) There is a coordinate system on $K$ such that (a) $K$ is a closed spherical neighborhood of $p$ in $K$ and (b) $G_{p}$ acts orthogonally.

(iv) The function $(g, x) \rightarrow g(x)$ defines a homeomorphism of $Q \times K$ onto a neighborhood of $p$.

Suppose that the theorem is false. Then there exists a point $z$ of $M$ such that every neighborhood of $z$ contains an infinite set $F$ such that, whenever $x, y \in F, G_{x}$ and $G_{y}$ are not conjugate. The totality of such points $z$ is clearly a closed subset $S$ of $M$ invariant under $G$. Let $S^{\prime}$ be the subset of $S$ consisting of all the points $z$ such that $\operatorname{dim} G_{z}$ $=\inf _{x \in S} \operatorname{dim} G_{x}$. Then there is a point $p$ of $S^{\prime}$ such that $G_{p}$ has the least number of components among those $G_{z}, z \in S^{\prime}$. Therefore for any $z$ in $S, G_{z}$ is not a proper subgroup of $G_{p}$.

Received by the editors December 3, 1955. 
According to (iii), there is a coordinate system $\left(x_{1}, \cdots, x_{n}\right)$ on $K$ with $p$ as its origin and such that (a) $K$ is given by $x_{1}^{2}+\cdots+x_{n}^{2} \leqq 1$ and (b) $G_{p}$ acts orthogonally. By (b), the fixed point set $A$ of $G_{p}$ in $K$ is linear and then it may be assumed to be

$$
x_{1}=\cdots=x_{r}=0, \quad x_{r+1}^{2}+\cdots+x_{n}^{2} \leqq 1 .
$$

From (ii), it is easily seen that whenever $x \in K, G_{x} \subset G_{p}$. Therefore $G_{x}=G_{p}$ for every $x \in A$.

Let $B$ be the $(r-1)$-sphere

$$
x_{1}^{2}+\cdots+x_{r}^{2}=1, \quad x_{r+1}=\cdots=x_{n}=0
$$

and let $f: K-A \rightarrow B$ be the map defined by

$$
\begin{aligned}
f\left(x_{1}, \cdots, x_{n}\right) & =\left(x_{1} / d, \cdots, x_{r} / d, 0, \cdots, 0\right), \\
d & =\left(x_{1}^{2}+\cdots+x_{r}^{2}\right)^{1 / 2} .
\end{aligned}
$$

For any $x \in K$, both $G_{x}$ and $G_{f(x)}$ are contained in $G_{p}$. It follows from (b) that $G_{x}=G_{f(x)}$. Since $p$ is a point of $S$ and $Q(K)$ is a neighborhood of $p$, there exists, by definition, an infinite set $F$ in $G(K)$ such that, whenever $x, y \in F, G_{x}$ and $G_{\nu}$ are not conjugate. Let $j$ be the projection of $Q(K)$ on $K$ (see (iv)). It is clear that for any $x \in Q(K), G_{j(x)}$ and $G_{x}$ are conjugate. Therefore $j(F)$ is an infinite set such that whenever $x, y \in j(F), G_{x}$ and $G_{y}$ are not conjugate. Since for every $x \in A, G_{x}=G_{p}$ and for every $x \in K-A, G_{x}=G_{f(x)}$, it follows that $F^{\prime}=f(j(F)-A)$ is an infinite set having the same property. $F^{\prime}$ is a subset of $B$ and then has a limit point $z$. By definition, $z$ belongs to $S$. Since $z \in K-A$, $G_{z}$ is a proper subgroup of $G_{p}$. This contradicts our choice of $p$. Hence the theorem is proved.

Corollary. Let $M$ and $G$ be as in the theorem. If $M$ is compact, then there exist a finite number of subgroups of $G$ such that the isotropic subgroup of $G$ at any point of $M$ is conjugate to one of them.

3. Now we are in a position to construct a noncompact analytic 3-manifold $M$ on which there is a circle group $G$ of analytic transformations such that every finite subgroup of $G$ is a $G_{x}$ for some $x$ in $M$. As mentioned above, this example is due to Professor Montgomery.

Roughly speaking, $M$ is obtained as follows: Cut a sequence of mutually disjoint open anchor rings off the euclidean 3-space, "twist" them and then piece them back to their complement. It is done in such a way that, if it is done for only one of these open anchor rings, the obtained space has a lens space as its one-point-compactification. 
We shall use the same symbol to denote a congruence class mod $2 \pi$ and any real number in this class. The additive group of congruence classes $\bmod 2 \pi$ will be denoted by $G$.

Let $(x, y, z)$ be coordinates in the euclidean 3 -space. For any positive integer $n, A_{n}$ denotes the open anchor ring

$$
\left(\left(x^{2}+y^{2}\right)^{1 / 2}-n\right)^{2}+z^{2}<(1 / 2)^{2}
$$

and $B_{n}$ the circle $x^{2}+y^{2}=n^{2}, z=0$. It is clear that every point of $A_{n}-B_{n}$ can be uniquely written

$$
((n+r \cos \beta) \cos \alpha,(n+r \cos \beta) \sin \alpha, r \sin \beta),
$$

which we shall abbreviate by $[r, \alpha, \beta]$, where $0<r<1 / 2$ and $\alpha, \beta \in G$. Let $f_{n}: A_{n} \rightarrow A_{n}$ be the function defined by

$f_{n} \mid B_{n}=$ identity;

$f_{n}[r, \alpha, \beta]=[r, \alpha-(n-1) \beta,-\alpha+n \beta]$ whenever $[r, \alpha, \beta] \in A_{n}-B_{n}$.

Then $f_{n}$ is one-one and onto (but not continuous at points of $B_{n}$ ) and $f_{n} \mid\left(A_{n}-B_{n}\right)$ is a homeomorphism.

The manifold $M$ to be constructed consists of the same points as the euclidean 3-space $E$. But it is topologized so that the natural map $j$ of $E-\bigcup_{n=1}^{\infty} B_{n}$ into $M$, defined by $j(x)=x$, and every map $f_{n}$ of $A_{n}$ (as a subspace of $E$ ) into $M$, defined as above, are open homeomorphisms into. $E-\cup_{n=1}^{\infty} B_{n}, A_{n}$ and $A_{n}-B_{n}$ are open subsets of $E$ and then inherit analytic structures from the natural one on $E$. Since $f_{n} \mid\left(A_{n}-B_{n}\right)$ is analytic for every $n$, we can easily have an analytic structure on $M$ such that $j$ and $f_{n}$ are all analytic. Hence $M$ is an analytic manifold.

For each $g \in G$, we define a transformation $g$ on $M$ such that $g \mid\left(M-\cup_{n=1}^{\infty} B_{n}\right)$ is a rotation of angle $g$ and each $g \mid B_{n}$ is a rotation of angle $n g$, where rotations are taken about the $z$-axis. In order to show that $g$ is analytic with respect to the analytic structure given above, we have only to show that $f_{n}^{-1} g f_{n}: A_{n} \rightarrow A_{n}$ is analytic for every $n$. Let the symbol $[r, \alpha, \beta]$ stand for

$$
((n+r \cos \beta) \cos \alpha,(n+r \cos \beta) \sin \alpha, r \sin \beta),
$$

where $0 \leqq r<1 / 2$ and $\alpha, \beta \in G$. Then

$$
\left(f_{n}^{-1} g f_{n}\right)[r, \alpha, \beta]=[r, \alpha+n g, \beta+g]
$$

and hence our assertion follows. This proves that $G$ acts analytically on $M$.

By construction, it is clear that whenever $x \in B_{n}, G_{x}$ is the cyclic subgroup of order $n$. Hence we have obtained an example as desired. 